\title{
Metastatic Spinal Cord Compression: A Prospective Observational Study at a Tertiary Care Center of North India
}

\author{
Rakesh Gocher, Pawan Kumar*, Shantanu Sharma, Rohitashwa Dana, Prashant Dadhich and Manish Kumar \\ Chaturvedi
}

Department of Radiation Oncology, SMS Medical College and Hospital, India

Submission: June 11, 2019; Published: July 15, 2019

*Correspondence Author: Pawan Kumar, Department of Radiation Oncology, SMS Medical College and Hospital, Jaipur, Rajasthan, India

Abstract

Introduction: Metastatic spinal cord compression (MSCC) is a most commonly encountered emergency in oncology, that can lead to permanent paraplegia if left untreated or treatment delayed, so it is essential to diagnose early and treat aggressively to preserve or improve the patient's quality of life. We analyzed the incident and the effect of common interventions on the outcome in these patients.

Material and Methods: We prospectively evaluated 80 patients of MSCC without any intervention in the year 2017 and collected all relevant patient and disease characteristics. All patients received corticosteroids. Seven patients were operated, 59 patients treated by radiotherapy and 3 patients were treated by surgery followed by radiotherapy.

Results: Most of the patients were in the age group of 40-70 years with male gender preponderance. Lung cancer was the most common incident (46.25\%) malignancy followed by breast, myeloma and prostate cancer. Dorsal spine was the most common site of compression (50.5\%) followed by lumbar spine (40.8\%) and cervical spine (8.7\%). 62 (77.5\%) patients were presented with pain at affected site and 60 (75\%) were presented with neurological motor deficit. $54(87 \%)$ patients got relieved from pain and only $23(38 \%)$ patients had motor improvement after treatment.

Conclusion: Patients presented 7-10 days after onset of neurological deficit were associated with poor outcome in neurological function, but radiotherapy proved an important modality for pain relief in MSCC and also beneficial in motor function preservation if instituted early. Ambulatory status of patient before starting the treatment was predictive of response to treatment and overall outcome of motor functions.

Keywords: Metastatic spinal cord compression; Paraplegia; Radiotherapy; Neurological motor deficit

\section{Introduction}

Metastatic spinal cord compression (MSCC) is an oncological emergency that occurs when cancer cells metastasize to the spine and then secondarily compresses the spinal cord. Although true incidence of spinal cord compression in cancer patients is unknown, it is estimated to develop in approximately $5 \%$ to $10 \%$ of all cancer patients $[1,2]$. Most of these patients are previously known cancer patients but it may be present, even without prior cancer diagnosis. The osseous vertebral column is affected in $85 \%$ of cases, paravertebral sites in $10 \%$ to $15 \%$, and there are rare cases of isolated epidural or intramedullary metastasis [3]. Back pain is the most common presenting symptom $(88 \%$ to $96 \%$ ), followed by weakness (76\% to $86 \%$ ), sensory deficits (51\% to $80 \%$ ), and autonomic dysfunction ( $40 \%$ to $64 \%$ ) $[4,5]$. Most common level of the metastatic spinal cord compression involvement is in the thoracic spine (59\% to $78 \%$ ), followed by lumbar $(16 \%$ to $33 \%)$ and cervical spine (4\% to $15 \%)$, while multiple levels are involved in up to half of patients $[4,6]$.

MSCC should be diagnosed early and immediate intervention required, if not treated immediately; virtually all of these patients would become paraplegic. Even with aggressive approach and therapy, the outcome of treatment is often being unsatisfactory and less than half of these patients are ambulatory and around $40 \%$ patients require permanent urinary catheterization. Although most patients with MSCC have limited survival, up to one third will survive beyond 1 year [7]. So, to prevent permanent paraplegia and preserve or improve the quality of life, it is essential to consider aggressive therapy. Motor function at presentation, rapidity of onset of symptom and type of histology are important prognostic factors for functional 


\section{Cancer Therapy \& Oncology International Journal}

outcome. Primary treatment is often selected depending on the patient's performance status, prognosis, and histological type of primary neoplasm. Most of these patients are not suitable for surgery. External beam radiotherapy has remained the mainstay of treatment in these patients. However, there is no clear consensus on the best radiotherapy dose and fractionation [8]. At our center, approximately seven thousand new cancer patients registered per year, and spinal cord compression is the most common oncological emergency in these patients.

\section{Material and Methods}

In year 2017 at Radiotherapy Department of SMS Hospital, Jaipur total 7656 new cancer patients registered out of which 98 patients were reported with metastatic spinal cord compression. However, only 80 patients were fulfilled the eligibility criteria for enrollment in this study. Less stringent eligibility criteria could have increased the enrollment (in some cases, there were clinical features of cord compression, but MRI scans were not available/ could not be done); hence, those patients were excluded from this study. So, prospectively 80 histological proven before or after cord compression and magnetic resonance imaging (MRI) documented spinal cord compression patient of malignancy were recruited. Patients were interviewed at the time of registration with a structured questionnaire including details of the occurrence and time of onset of back pain, paraparesis, weakness, and bladder/ bowel dysfunction. The date of onset of symptoms of spinal cord compression was recorded by history taking supplemented by cross checking all patient records. Delays were expressed in terms of whole days. Neurological status was documented when the patients reported to our outpatient department and after completion of treatment and graded as follows: Motor function (0 - no contraction; 1 - flicker or trace of contraction; 2 - active movement possible only with gravity eliminated; 3 - active movements against gravity but not resistance; 4 - active movement against resistance and gravity; and 5 - normal powers). Sensory symptoms and signs along with bladder and bowel function were also recorded before and

after completion of treatment. All data related to patients were well maintained in the files at hospital-based cancer registry (HBCR). All patients received corticosteroids in the form of dexamethasone (16-24 mg in divided doses) as soon as possible.

All patients were assessed jointly by oncologists and neurosurgeons in the hospital for feasibility of surgery for rapid decompression. Radiation was delivered by four protocols

a) 4 patients received 40 Gy in 20 fractions @ 2Gy per fraction over 4 weeks

b) 34 patients received 30 Gy in 10 fractions @ 3Gy per fraction over 2 weeks

c) 12 patients received 20 Gy in 5 fractions @ 4Gy per fraction over 1 week

d) 12 patients received 25 Gy in 4 fractions @ 6.25Gy per fraction every week

Depending on the overall assessment of the patient and expected outcome. Assessment of pain was done by adult nonverbal pain (ANVP) scale given by The University of Rochester Medical Center before starting the treatment and after completion of the treatment and documented the scale on 0 -10 scale.

\section{Results}

Table 1: Age and gender characteristics $(n=80)$.

\begin{tabular}{|c|c|c|c|}
\hline Age & Number of patients (\%) & Male & Female \\
\hline$\leq 20$ & $5(6.25)$ & 3 & 2 \\
\hline $21-30$ & $3(3.75)$ & 1 & 2 \\
\hline $31-40$ & $6(7.5)$ & 3 & 3 \\
\hline $41-50$ & $14(17.5)$ & 8 & 6 \\
\hline $51-60$ & $14(17.5)$ & 9 & 5 \\
\hline $61-70$ & $22(27.5)$ & 13 & 9 \\
\hline$\geq 71$ & $16(20.0)$ & 11 & 5 \\
\hline Total & $80(100)$ & $48(60 \%)$ & $32(40 \%)$ \\
\hline
\end{tabular}

Table 2: Site of primary tumor $(n=80)$ and level of cord compression $(n=103)$.

\begin{tabular}{|c|c|c|c|c|}
\hline \multirow{2}{*}{ Primary site } & \multirow{2}{*}{ Number of patients (\%) } & \multicolumn{3}{|c|}{ Number of Compression events at Spine Level } \\
\cline { 3 - 5 } & & Cervical & Dorsal & Lumbar \\
\hline Lung & $37(46.25)$ & 4 & 27 & 5 \\
\hline Multiple Myeloma & $8(10)$ & 2 & 3 & 3 \\
\hline Unknown Primary & $6(7.5)$ & 1 & 3 & 4 \\
\hline Prostate & $6(7.5)$ & 1 & 2 & 0 \\
\hline Esophagus & $2(2.5)$ & 0 & 2 & 2 \\
\hline Lymphoma & $3(3.75)$ & 0 & 1 & 3 \\
\hline Breast & $9(11.25)$ & 1 & 1 & 1 \\
\hline Choriocarcinoma & $1(1.25)$ & 0 & 1 & 0 \\
\hline Ewing Sarcoma & $2(2.5)$ & 0 & 0 & 1 \\
\hline Schwannoma & $1(1.25)$ & 0 & & \\
\hline Kidney & $1(1.25)$ & & & \\
\hline
\end{tabular}




\section{Cancer Therapy \& Oncology International Journal}

\begin{tabular}{|c|c|c|c|c|}
\hline Buccal Mucosa & $1(1.25)$ & 0 & 1 & 0 \\
\hline Cervix & $1(1.25)$ & 0 & 1 & 1 \\
\hline Tongue & $1(1.25)$ & 0 & 0 & 0 \\
\hline Urinary Bladder & $1(1.25)$ & 0 & $* 52(50.5 \%)$ & $* 42(40.8 \%)$ \\
\hline Total & $80(100)$ & $* 9(8.7 \%)$ & 0 & 1 \\
\hline
\end{tabular}

*23 patients had two sites of compression of spinal cord; therefore, compression events are 103 and patient number is 80 .

Most of the patients were in the age group of 41-70 years and male female ratio was 3:2 (Table 1). There was no direct relation between primary malignancy and the site of cord compression. $24(30 \%)$ patients had synchronous metastasis in liver, lung, adrenal, brain and bone at the time of cord compression. Solitary bone lesion causing cord compression was found in 31 (38.7\%) patients. Most commonly presented primary was Lung cancer (46.25\%) to cause spinal cord compression which was followed by female breast cancer, multiple myeloma, and prostate cancer. Spinal cord compression presented as metastasis of unknown origin in $9(11.25 \%)$ patients. Other least common causes included 3 patients of lymphoma (3.75\%), 2 patients of esophageal cancer $(2.5 \%), 2$ patients of Ewing sarcoma $(2.5 \%)$ and1 patient from each of choriocarcinoma, schawnomma, kidney, buccal mucosa, cervix, tongue and urinary bladder carcinoma (Table 2).

Table 3: Onset of cord compression after diagnosis of malignancy $(n=80)$.

\begin{tabular}{|c|c|}
\hline Onset of cord compression (months) & Number of patients (\%) \\
\hline At presentation & $36(45)$ \\
\hline$<12$ & $26(32.5)$ \\
\hline $13-24$ & $12(15)$ \\
\hline $25-36$ & $4(5)$ \\
\hline $37-48$ & $2(2.5)$ \\
\hline $49-60$ & $0(0)$ \\
\hline$>60$ & $0(0)$ \\
\hline
\end{tabular}

Lumbar spine was the most common site of compression (40.7\%) followed by upper dorsal $(21.3 \%)$ and lower dorsal (15.5\%) spine (Table 2). 57 (71\%) patients had a single level of cord compression and 23 (29\%) patients presented with multiple levels of cord compression, usually a combination of lower dorsal and lumbar spine. 36 (45\%) patients initial presentation was with spinal cord compression (among other symptoms) and among these, in $9(11.25 \%)$ patients, primary site of malignancy could not be located with routine diagnostic tests (Table 3). Most of the compression events occurred within 2 years of diagnosis. Most patients presented to our hospital after mean delay of around 15 days from the onset of symptoms with a range of 1-60 days. 62 patients (77.5\%) presented with pain localized to the site of metastasis and only 15 (18.75\%) patients presented with weakness of limbs without pain. Complete paraplegia was present in $6(7.5 \%)$ of patients (Table 4$)$. Sensory loss was present in only $16(20 \%)$ and bladder involvement in $18(22.5 \%)$ patients at presentation. All patients received corticosteroids
(16-24 mg in divided doses). A rapid decompression was done only in 7 (8.75\%) patients in Neurosurgery Department. Radiotherapy was delivered in $62(77.5 \%)$ patients which also includes 3 patients came from neurosurgery department after surgical decompression. 11 patients were treated only by symptomatic measures due to poor prognostic features and low performance score.

Table 4: Motor deficit of patients at presentation $(n=80)$.

\begin{tabular}{|c|c|}
\hline $\begin{array}{c}\text { Motor deficit (grade) at } \\
\text { presentation }\end{array}$ & Number of patients (\%) \\
\hline 0 & $6(7.5)$ \\
\hline 1 & $10(12.5)$ \\
\hline 2 & $10(12.5)$ \\
\hline 3 & $16(20)$ \\
\hline 4 & $18(22.5)$ \\
\hline 5 & $20(25)$ \\
\hline
\end{tabular}

Three patients defaulted (Table 5). Of 62 patients who received radiotherapy, only 23 (38\%) patients had motor improvement ( 2 patients out of 4 from 40 gray 20 fraction arm, 9 patients out of 34 from 30 gray 10 fractions arm, 5 patients out of 12 from 20 gray 5 fraction arm and 4 patients out of 12 from 25 gray 4 fraction arm). Head to head comparison was not done in between all these used fractionation schedules due to different patient selection criteria for each schedule and different number of patients in each schedule. An analysis of these patients revealed that there was complete recovery of power in only 12 patients, and further analysis revealed that all these patients had either grade 3 or grade 4 power before starting the treatment. A maximum net gain of motor function was three grades of power and was found in 3 patients.

Table 5: Treatment received by patients $(n=80)$.

\begin{tabular}{|c|c|}
\hline Treatment Received & Number of patients \\
\hline \multicolumn{2}{|c|}{ Surgery } \\
\hline $\begin{array}{l}\text { Decompression with } \\
\text { laminectomy }\end{array}$ & 5 \\
\hline Decompression with fixation & 2 \\
\hline \multicolumn{2}{|c|}{ Radiotherapy (gray/fraction) } \\
\hline $25 / 4$ & 12 \\
\hline $30 / 10$ & 34 \\
\hline $20 / 5$ & 12 \\
\hline $40 / 20$ & 4 \\
\hline No treatment planned & 11 \\
\hline Defaulted & 3 \\
\hline
\end{tabular}


In other words, no patient with Grade 0 or 1 power recovered fully for motor and sensory function but they responded well for pain. There was no improvement in 16 patients and deterioration was recorded in 6 patients. Thus, grade of power before starting the treatment was predictive of response to treatment and overall outcome of motor or sensory functions. Delay of treatment by more than 7 days was associated with poor outcome in terms of neurological function. Assessment of response in pain was made according ANVP scale. Response in terms of pain relief recorded in 54 patients out of 62 patients who are presented with pain at metastatic site. 6 patients recorded no improvement on pain scale after radiotherapy and 2 patients recorded worsening of pain during the treatment. Maximum improvement noted at ANVP scale was 6 in 24 patents and improvement of 2 to 5 were noted in 30 patients.

\section{Discussion}

Metastatic Spinal cord compression is an oncological emergency which can lead to permanent neurologic impairment that can lead to poor quality of life if not treated early. Metastatic spinal cord compression should be suspected in any patient who presents with progressively increasing back pain, urinary incontinence, paraparesis or paraplegia, mainly in the high-risk population such as long-time smokers with history of chronic cough or women with a family history of breast cancer [1]. In a review report by Fuller and colleagues of over 1000 patients with metastatic spinal cord compression, the most common primary tumor type is breast cancer (29\%), followed by lung cancer $(17 \%)$ and prostate cancer $(14 \%)$, which reflects the high natural incidence of these tumors [6]. New-onset back pain in cancer patients needs to be taken very seriously and proper evaluation must be carried out.

So, it is essential to diagnose early and treat aggressively [9]. Ambulatory status at the time of diagnosis has been shown to be of high favorable prognostic significance with regards to the outcome of treatment of MSCC, as is a slower development of motor deficit [10]. However, most common reason of failure is late reporting to the appropriate facility or land up with permanent damage of spinal cord. Surgery and Radiotherapy are the available options for treatment of MSCC. However, most of the patients with MSCC are unfit to undergo surgery; radiotherapy is the most commonly used option. In general, primary tumours that are very sensitive to radiotherapy are lymphomas, myeloma and seminomatous germ cell tumours. Most solid tumours, such as breast cancer, prostate cancer and lung cancer, are considered to have intermediate radiosensitivity. Melanomas, osteosarcomas and renal cell carcinomas are usually considered to be more radioresistant $[11,12]$.

There was so many data published on MSCC, but the best recommended radiotherapy dose and fractionation schedule for MSCC yet to be defined. The choice of dose and fractionation schedule currently depends on multiple factors, which include the primary site of the malignancy; the interval between tumour diagnosis and the development of cord compression; other extensive bone or visceral metastases at the time of radiotherapy; ambulatory status and the length of time for developing motor deficits before treatment; life expectancy at the time of treatment and history of previous radiotherapy are few of them [13]. Palliative radiotherapy has been the standard of care in the treatment of patients with MSCC. Many dosing schedules are currently in practice, and it varies according to institutional protocols, although a total of $30 \mathrm{~Gy}$ in 10 fractions is the most frequently employed fractionation schedule. Guidelines from the Royal College of Radiologists suggest that a single 8 Gy dose to those with a life expectancy of fewer than 6 months with established neurological deficit is appropriate and 20 Gy in five fractions or $30 \mathrm{~Gy}$ in 10 fractions to others with a good prognosis [14].

Another important trial by Rades et al. compared 30 Gy in 10 fractions versus $40 \mathrm{~Gy}$ in 20 fractions in a total of 214 patients and analyzes both functional outcome and ambulatory status. The study showed no detriment to functional outcome with the shorter 10 fraction regimen, yet with obvious benefit in terms of resources, machine time and costs [15].

In a retrospective series by Rades et al. reported 1,304 patients with MSCC were separated into five schedules: $8 \mathrm{~Gy} \times$ 1 in 1 day ( $n=261$, group 1$), 4$ Gy $\times 5$ in 1 week $(n=279$, group 2), 3 Gy $\times 10$ in 2 weeks ( $=274$, group 3$), 2.5$ Gy $\times 15$ in 3 weeks ( $\mathrm{n}=233$, group 4), and 2 Gy $\times 20$ in 4 weeks ( $=257$, group 5). All the groups had similar post treatment ambulatory rates $(63 \%$ to $74 \%)$ and motor functions improvement $(26 \%$ to $31 \%)$. However, in-field recurrence rates were much lower for the protracted schedules. The 2-year in-field recurrence rates for groups $1,2,3,4$, and 5 were $24 \%, 26 \%, 14 \%, 9 \%$, and $7 \%$ $(\mathrm{P}<.001)$, respectively. They recommend that a single fraction of 8 Gy should be used in MSCC patients with limited survival expectations and that 30 Gy in 10 fractions should be used for all other patients [16].

At our institute we preferred weekly 6.25 Gy single fractions for four weeks that delivers total dose of 25 Gy as hypo fractionated schedule. Biological Equivalent Dose (BED) of this schedule (40.6) is almost similar with 30 Gy in 10 fractions (39 Gy). We observed that weekly hypo fractionated schedule is a feasible modality for patient as well as for a treatment center with high patient load. This modality was well tolerated by all the patients and results were also comparable with commonly used schedule of 30Gy in 10 fractions. In breast cancer patients only, bony metastasis has a better prognosis and visceral metastasis resulted in worse outcome. Median survival after spinal cord compression is better in radiosensitive primary malignancy (like Multiple myeloma, Breast cancer, prostate cancer etc.) with a single metastasis. Patients with multiple metastases, visceral or brain metastases, and lung or gastrointestinal cancers have the shortest survival $[17,18]$. 
In this study, we prospectively analyze the clinical features and treatment outcomes of spinal cord compression due to malignancy. Most compression events occurred within first 2 years of diagnosis. Metastatic cord compression was most commonly found in lung cancer $(46.25 \%)$, breast cancer (11.25\%), myeloma (10\%), and prostate cancer (7.5\%). According to HBCR of 2017 lung cancer is one of the most commonly reported malignancy after head and neck malignancy and breast cancer is one of the most common encountered malignancy in females. So, distribution of cord compression among various malignancies correlated with the most incident cancers that were registered at our HBCR. Most of the patients were in the age group of 40-70 years and slightly male gender preponderance in patients that was most probably because of higher incident of lung cancer cases in male population.

Dorsal spine was the most common site of compression (50.5\%) followed by lumbar spine (40.8\%) and only $8.7 \%$ had compression in cervical spine. The high incidence of compression along dorsal and lumbar regions may be due to the high incidence of metastasis at these sites and/or due to progressively increasing weight bearing craniocaudally and subsequent fracture of vertebrae. In 23 (28.7\%) patients, there was a synchronous second site of cord compression and in 28 (35\%) patients a synchronous metastasis to liver, lung, brain, and/or other site were present at the time of cord compression. Solitary bone lesion causing cord compression was found in 29 $(36.25 \%)$ patients. Most patients $(77.5 \%)$ presented with pain localized to the site of metastasis, $60(75 \%)$ patients presented with neurological motor deficit and only 15 (18.75\%) patients presented with weakness of limbs that was not associated with pain.

All patients in our study received high dose dexamethasone as early as possible with gastro protection by proton pump inhibitors with a high degree of suspicion or immediately after the diagnosis of cord compression. The primary objective of corticosteroid therapy is reduction of edema and inflammation at the site of spinal cord compression and they serve as an effective bridge to definitive therapy [1]. A very good response observed in terms of pain relief in $54(87 \%)$ patients, but only 23 (38\%) patients had motor improvement after treatment. Though metastatic spinal cord compression is a well-known medical emergency, but we observed that most of patients presented late from the onset of symptoms with mean delay of 7-10 days. Spinal cord compression symptom may be an initial presentation of malignancy. In our study 36 (45\%) patients had been presented with chief complain of cord compression symptoms without prior diagnosed malignancy and we were fail to locate the site of primary tumor in $6(7.5 \%)$ patients with routine available diagnostic tests. Radiotherapy was delivered through four protocols depending upon the performance status, life expectancy, metastatic status of disease and expected outcome in neurological function.
In majority of patients, we used 30 gray in 10 fractions, and response rate was almost similar with all used fractionated schedule. We observed that complete or near complete motor response were observed only in early reported cases (within 7-10 days after onset of cord compression symptoms) and thereafter all patients were satisfied only with pain relief or some grade of motor improvement or no improvement. So, there should be a high index of suspicion for MSCC in known case of malignancy who presented with back pain which had to be diagnosed early and treated as soon as possible. Neurosurgical intervention was done only in 7 ( $8.75 \%)$ patients in whom cord compression was a presenting symptom. Many of the patients were not fit for surgery or not willing to undergo surgical trauma. So, there was a general tendency of underutilization of surgical intervention when patients had an established malignancy.

\section{Conclusion}

Patient presented 7-10 days after onset of neurological deficit was associated with poor outcome in neurological function, but radiotherapy proved an important modality for pain relief in MSCC and also beneficial in motor function preservation if instituted early. Ambulatory status before starting the treatment was predictive of response to treatment and overall outcome of motor functions. High index of suspicion, early diagnosis and early intervention is the key steps to achieve a better quality of life in MSCC patients. Better results can be obtained by sensitizing high risk patients and their family members and Research is needed to develop a standard management protocol for MSCC cases. Even though palliative radiotherapy has been the standard of care in the treatment of patient with MSCC but there was not any standard management protocol till date in literatures. Weekly hypo fractionated schedule of 6.25 Gy per fraction may be a better alternative option for high patient load centers.

\section{References}

1. Kwok Y, Tibbs PA, Patchell RA (2006) Clinical approach to metastatic epidural spinal cord compression. Hematol Oncol Clin North Am 20(6): 1297-1305.

2. Byrne TN (1992) Spinal cord compression from epidural metastases. N Engl J Med 327: 614-9.

3. Gerszten PC, Welch WC (2000) Current surgical management of metastatic spinal disease. Oncology 14: 1013-1026.

4. Gilbert RW, Kim JH, Posner JB (1978) Epidural spinal cord compression from metastatic tumor: diagnosis and treatment. Ann Neurol $3(1): 40-51$.

5. Heldmann U, Myschetzky PS, Thomsen HS (1997) Frequency of unexpected multifocal metastasis in patients with acute spinal cord compression. Evaluation by low-field MR imaging in cancer patients. Acta Radiol 38(3): 372-375.

6. Fuller BG, Heiss JD, Oldfield EH (2001) Spinal cord compression. In: DeVita VT, Hellman S, Rosenberg SA, editors. Cancer: principles and practice of oncology. Philadelphia (PA): Lippincott Williams and Wilkins p 2617-2633. 


\section{Cancer Therapy \& Oncology International Journal}

7. Maranzano E, Latini P, Checcaglini F (1991) Radiation therapy in metastatic spinal cord compression: a prospective analysis of 105 consecutive patients. Cancer 67: 1311-1317.

8. Prewett S, Venkitaraman R (2010) Metastatic spinal cord compression: Review of the evidence for a radiotherapy dose fractionation schedule. Clin Oncol (R Coll Radiol) 22(3): 222-230.

9. Higdon ML, Higdon JA (2006) Treatment of oncologic emergencies. Am Fam Physician 74: 1873-1880.

10. Rades D, Fehlauer F, Veninga T (2007) Functional outcome and survival after radiotherapy of metastatic spinal cord compression in patients with cancer of unknown primary. Int J Radiat Oncol Biol Phys 67(2) 532-537.

11. Loblaw DA, Laperriere NJ (1998) Emergency treatment of malignant extradural spinal cord compression: an evidence-based guideline. J Clin Oncol 16(4): 1613-1624

12. Levack P (2001) A prospective audit of the diagnosis, management and outcome of malignant cord compression. 97/08. London: CRAG, NICE Clinical Audit \& Resource Group.
13. Rades D, Dunst J, Schild SE (2008) The first score predicting overall survival in patients with metastatic spinal cord compression. Cancer 112(1): 157-161.

14. Board of Faculty of Clinical Oncology (2006) Radiotherapy dose fractionation. Royal College of Radiologists, London, UK.

15. Rades D, Fehlauer F, Stalpers LJ (2004) A prospective evaluation of two radiotherapy schedules with 10 versus 20 fractions for the treatment of metastatic spinal cord compression: final results of a multicenter study. Cancer 101(11): 2687-2692.

16. Rades D, Stalpers LJ, Veninga T (2005) Evaluation of five radiation schedules and prognostic factors for metastatic spinal cord compression. J Clin Oncol 23(15): 3366-3375.

17. Sherry MM, Greco FA, Johnson DH, Hainsworth JD (1986) Breast cancer with skeletal metastases at initial diagnosis. Distinctive clinical characteristics and favorable prognosis. Cancer 58: 178-182.

18. Helweg-Larsen S, Sørensen PS, Kreiner S (2000) Prognostic factors in metastatic spinal cord compression: A prospective study using multivariate analysis of variables influencing survival and gait function in 153 patients. Int J Radiat Oncol Biol Phys 46(5): 1163-1169.

\section{Your next submission with Juniper Publishers will reach you the below assets}

- Quality Editorial service

- Swift Peer Review

- Reprints availability

- E-prints Service

- Manuscript Podcast for convenient understanding

- Global attainment for your research

- Manuscript accessibility in different formats

( Pdf, E-pub, Full Text, Audio)

- Unceasing customer service

Track the below URL for one-step submission https://juniperpublishers.com/online-submission.php 